\title{
Ensino Híbrido: Relato de Experiência sobre o uso de AVEA em uma proposta de Sala de Aula Invertida para o Ensino Médio.
}

\author{
Hybrid Teaching: Integration of Virtual Environment of Teaching and Learning to a \\ proposal of Flipped Classroom for High School.
}

\author{
Aline Coêlho dos Santos ${ }^{1}$ \\ Programa de Pós-Graduação em Tecnologias da Informação e Comunicação/ UFSC \\ Priscila Cadorin Nicolete ${ }^{2}$ \\ Programa de Pós-Graduação em Informática na Educação/ UFRGS \\ Natália Mattiola ${ }^{3}$ \\ Programa de Pós-Graduação em Tecnologias da Informação e Comunicação/ UFSC \\ Juarez Bento da Silva ${ }^{4}$ \\ Programa de Pós-Graduação em Tecnologias da Informação e Comunicação/ UFSC
}

\begin{abstract}
RESUMO: Este artigo trata de um relato de experiência sobre integração de tecnologias educacionais numa proposta de sala de aula invertida, aplicada nas aulas de Biologia, em uma turma de Ensino Médio. Pretende-se com esse estudo discutir sobre os recursos tecnológicos e metodologia de aprendizagem utilizados nos processos de planejamento e execução das aulas. Para seu desenvolvimento, foi construído um curso on-line em Ambiente Virtual de Ensino e Aprendizagem, sobre o conteúdo de fungos, logo ocorreu a aplicação em sala de aula da ferramenta. Em sequência, foi aplicado aos alunos questionário aberto sobre os pontos positivos e negativos encontrados frente ao uso da ferramenta, possibilitando um breve relato sobre a experiência dos mesmos ao utilizarem esta tecnologia em sala de aula. Por fim, identificou-se que a proposta trazida pelo Ensino Híbrido, em que "misturamos" metodologias de aprendizagem ativa com tecnologias educacionais tende a gerar bons resultados para o aprendizado do aluno, pois aumenta consideravelmente a qualidade de estudo do aluno, e o nível de discussão em sala de aula.
\end{abstract}

Palavras-chave: Sala de Aula Invertida; Ensino Híbrido; Tecnologias Educacionais.

\begin{abstract}
This article is about an experience report about the integration of educational technologies into an inverted classroom proposal applied in biology classes in a high school class. The aim of this study is to discuss the technological resources and learning methodology used in the planning and execution of the classes. For its development, an online course was created in Virtual Environment of Teaching and Learning, on the fungi content, soon occurred the application in the classroom of the tool. In the sequence, the students were asked an open questionnaire about the positive and negative points found in relation to the use of the tool, allowing a brief report about their experience in using this technology in the classroom. Finally, it was identified that the proposal brought by Hybrid Teaching, in which "we mix" active learning methodologies with educational technologies tends to generate good results for student learning, as it considerably increases the quality of student study, and the level of discussion in the classroom.
\end{abstract}

Keywords: Flipped Classroom; Hybrid Teaching; Educational Technologies. 


\section{Introdução}

Há anos discute-se, não só no Brasil, mas em todo mundo, meios que nos permitam ofertar ensino de qualidade nas escolas de Educação Básica. No entanto, essas discussões tornaram-se ainda mais latentes com a chegada das Tecnologias Digitais da Informação e Comunicação (TDIC) nos espaços escolares, pois entendia-se naquele momento que muitas formas de ensinar não se justificariam mais (MORAN, 2000). Essas tecnologias não mudaram apenas as formas de produção, organização e difusão da informação, mas a maneira como enxergamos e entendemos o mundo, ou seja, atingiu diretamente nossa cultura, e modificou rapidamente nossa forma de aprender, confrontando todo o sistema de ensino, há anos consolidado (KENSKI, 2012).

Diante desse cenário, o ensino híbrido surge, na atualidade, como uma alternativa de inovar no processo de ensino-aprendizagem por meio de uma abordagem pedagógica que "mistura" atividades presenciais com atividades viabilizadas por meio das tecnologias educacionais, tornando o processo de aprender mais atrativo e dinâmico (MORAN, 2015).

De acordo com essa abordagem, o conteúdo e as instruções sobre um determinado assunto curricular não são transmitidos pelo professor em sala de aula. $\mathrm{O}$ aluno estuda o material em diferentes situações e ambiente, e a sala de aula passa a ser um lugar de aprender ativamente, realizando atividades de resolução de problemas ou projetos, discussões, laboratórios entre outros, com o apoio do professor e colaborativamente com os colegas (VALENTE, 2015 apud BACICH, NETO \& DE MELLO, 2015, p. 9).

Valente (2014) propõe que essa integração tem viabilizado a oferta do ensino híbrido (em inglês conhecido como blended learning), sendo a sala de aula invertida (em inglês flipped classroom), um exemplo clássico dessa abordagem, que vem sendo fortemente incorporada em diferentes níveis de ensino, inclusive no que tange a Educação Básica.

Dentro desse contexto, esse estudo preocupa-se em relatar e discutir sobre o processo de construção e aplicação de uma aula sobre Fungos, realizada em uma turma de Ensino Médio, na disciplina de Biologia, planejada conforme direcionamentos apontados pelo ensino híbrido, integrando Ambiente Virtual de Ensino e Aprendizagem (AVEA) e uma das metodologias de aprendizagem ativa, conhecida como sala de aula invertida.

\section{Embasamento Teórico}

Esse estudo está pautado sob duas variáveis, são elas: Sala de Aula Invertida e Ambiente Virtual de Ensino e Aprendizagem, que se integram nesse trabalho, enquanto exemplo de ensino híbrido.

Sala de aula invertida é um dos tipos de metodologia de aprendizagem ativa, que embora discutida desde a década de XX, tem se fortalecido na atualidade por conta da difusão das TDIC nos ambientes escolares (SCHMITZ, 2016). Moran (2014) afirma que esta metodologia é a que permite maior flexibilização para a integração de tecnologia ao processo de ensino-aprendizagem, pois deixa a cargo do virtual a disponibilização do material necessário ao estudo prévio, e em sala de aula, propõe atividades mais dinâmicas e criativas, podendo dessa forma combinar diferentes formas de aprender por meio de recursos diversos. 
Nesse sentido, Schmitz (2016) define três momentos para elaboração do plano de aula "invertida", são eles: (i) o momento antes da aula, onde o professor prepara o conteúdo, diponibiliza em AVEA para o aluno, e este realiza antecipadamente seus estudos; (ii) o momento durante a aula, onde o aluno realiza atividades práticas e retira dúvidas; (iii) o momento depois da aula, onde é feito o fechamento do conteúdo proposto, avaliando-o, identificando a necessidade de novos estudos, e compartilhando as informações adquiridas. Sendo assim, os AVEA são ferramentas eletrônicas que podem tornar o processo de ensino-aprendizagem mais ativo, dinâmico e personalizado, pois as mídias incorporadas a ele promovem interatividade e colaboração entre professores, alunos e conhecimentos explorados (PEREIRA; SCHMITT; DIAS, 2007).

Muito difundido atualmente, o Moodle, é um exemplo de AVEA, que foi projetado para ser um sistema muito simples de ser acessado e personalizado, compatível com diferentes dispositivos, e que possibilita a criação de um ambiente colaborativo, com aprendizagem centrada no aluno (DOUGIAMAS; TAYLOR, 2009).

Como um espaço de ensino-aprendizagem, o Moodle, fornece muitas ferramentas, como: (a) fóruns de discussão configuráveis; (b) gestão de conteúdos; (c) criação de diferentes tipos de questionários; (d) sistema de chat com registo de histórico configurável; (e) editor Wiki; (f) sistema de distribuição de inquéritos estandardizados; (g) sistema de gestão de tarefas, entre outros recursos facilmente administráveis pelo professor e manuseáveis pelo aluno (VALENTE; MOREIRA, 2007).

Nesse contexto, é perceptível diante das potencialidades apresentadas pelo Moodle, que há uma a forte relação entre AVEA com promoção de aprendizagem ativa, pois este possibilita a personalização do ensino numa perspectiva de tornar o aluno produtor do conhecimento, dando a ele autonomia e flexibilização de tempo e espaço para essa produção.

\section{Metodologia}

Este artigo está pautado na descrição e discussão de uma aula desenvolvida sob as diretrizes proposta pela metodologia de sala de aula invertida, utilizando como recurso viabilizador o AVEA - Moodle, disponibilizado pela plataforma on-line InTecEdu, que integra projetos de pesquisa e extensão do Laboratório de Experimentação Remota (RexLab) da Universidade Federal de Santa Catarina (UFSC), campus Araranguá/SC. Sendo assim, este estudo caracteriza-se como qualitativo de caráter descritivo, pois apresenta, segundo Gil (1991), "diferentes interpretações que partem da visão de mundo do indivíduo", o que possibilita uma análise cautelosa centrada no objeto de estudo através da análise de dados indutiva e levantamento de dados a seu respeito. Para efetivação dessa pesquisa, foi necessária a conclusão das seguintes etapas: (i) o planejamento da aula "invertida" por meio da elaboração de um plano de aula detalhado; (ii) escolha e preparação dos materiais e atividades a serem exploradas pelos alunos; (iii) construção de curso no Moodle para veiculação dos materiais e atividades propostas; (iv) aplicação da aula sobre fungo numa abordagem de Sala de Aula Invertida; (v) aplicação de questionário aberto, a fim de identificar a experiência dos alunos por meio dos pontos positivos e negativos elencados por eles; (vi) produção desse relato.

\subsection{Planejamento da aula}

No planejamento das aulas sobre fungos realizou-se a construção de um plano de aula detalhado, a organização do material didático a ser utilizado e a 
elaboração das atividades propostas nele. Dentro desse escopo estipulou-se: (a) o conteúdo a ser explorado; (b) a abordagem metodológica empregada; (c) o público-alvo; (d) a duração; (e) os objetivos de aprendizagem; (f) as estratégias didáticas; (g) os recursos; e (h) as formas de avaliação.

Dessa forma, ficou definida, a construção de um plano de aula sobre fungos, numa abordagem de sala de aula invertida, organizada em 6 encontros, sendo 3 presenciais e 3 a distância, com data inicial prevista para o dia 20 de março de 2017 e data final para o dia 17 de abril de 2017. As estratégias de aula foram direcionadas para turmas de Ensino Médio, com previsão de aplicação em uma turma do $4^{\circ}$ ano do Ensino Médio Integrado do Instituto Federal de Santa Catarina, campus Araranguá, composta por 30 alunos, sendo 1 menino e 29 meninas, com faixa-etária entre 15 e 18 anos.

Quanto aos recursos, além dos materiais organizados e elaborados pelo professor titular da disciplina, ficou estabelecido o uso de laboratório de informática, AVEA - Moodle, projetor e internet. Em relação a organização do trabalho pedagógico, a mesma pode ser observada, resumidamente, na tabela 1 .

Tabela 1 - Organização do trabalho pedagógico

\begin{tabular}{clll}
\hline Encontro & Modalidade & & \multicolumn{1}{c}{ Estratégias (Encaminhamentos) } \\
\hline $\mathbf{1}$ & Presencial & a) & Aula expositiva conceitual sobre características gerais dos fungos; \\
& & b) & Apresentação da plataforma InTecEdu; \\
& c) & Explicaçães sobre as formas de acesso e matrícula ao curso; \\
& & d) & Apresentação de Materiais para estudo, já disponíveis no AVEA. \\
\hline $\mathbf{2}$ & À Distância & e) & Realização de matrícula ao curso; \\
& & f) & Exploração de materiais disponibilizados na seção "Antes" do curso; \\
& g) & Realização de estudo prévio dos materiais. \\
\hline $\mathbf{3}$ & Presencial & h) & Atividade em grupo; \\
& i) & Realização de atividades propostas na seção "Durante". \\
\hline $\mathbf{4}$ & À Distância & j) & $\begin{array}{l}\text { Realização de avaliação conceitual sobre fungos, disposta na seção "Depois" } \\
\text { do curso; }\end{array}$ \\
\hline $\mathbf{5}$ & Presencial & k) & $\begin{array}{l}\text { Apresentação de seminário de pesquisa para socialização dos trabalhos } \\
\text { desenvolvidos; }\end{array}$ \\
\hline $\mathbf{6}$ & À Distância & 1) & $\begin{array}{l}\text { Elaboração de considerações sobre as aulas planejadas para o conteúdo de } \\
\text { fungos, apontando no AVEA, os pontos positivos e negativos referentes à } \\
\text { experiência vivenciada. }\end{array}$ \\
\hline & &
\end{tabular}

Fonte: Elaborado pelos autores, 2017

Quanto às formas de avaliação ficaram previstas 5 avaliações distintas, representadas na tabela 2, valorizando as diferentes habilidades e competências apresentadas pela diversidade de perfis que se tem em uma mesma turma. Dessa maneira optou-se por uma avaliação processual, contínua e diversificada, que conforme o Plano de Desenvolvimento Institucional (PDI) e Projeto Pedagógico de Curso (PPC), para as turmas de Ensino Médio Integrado em Vestuário, do Instituto Federal de Santa Catarina (IFSC), indica avanços e dificuldades na ação educativa, remetendo o professor a uma reflexão sobre a prática pedagógica (PDI, 2015; PPC, 2016).

Tabela 2 - Avaliações previstas para conteúdo de fungos.

\begin{tabular}{cl}
\hline \multicolumn{1}{c}{ Atividades Avaliativas } \\
\hline I. & $\begin{array}{l}\text { Construção de Mapa Conceitual, parte da atividade proposta na seção "durante”, realizada } \\
\text { presencialmente. }\end{array}$ \\
\hline II. & $\begin{array}{l}\text { Atividade de pesquisa, referente a conclusão da atividade proposta na seção "durante”, realizada na } \\
\text { modalidade à distância. }\end{array}$ \\
\hline III. & $\begin{array}{l}\text { Seminário de Pesquisa, compreendendo a exposição e compartilhamento de resultados obtidos nas } \\
\text { atividades concluídas, efetivadas em grupo. }\end{array}$ \\
\hline IV. & Avaliação Conceitual, \\
\hline V. & $\begin{array}{l}\text { Avaliação atitudinal, considerando o envolvimento e responsabilidade durante todo processo de ensino, } \\
\text { incluindo: (a) efetivação de matrícula previamente; (b) realização de estudo prévio; (c) envolvimento no } \\
\text { trabalho em grupo; (d) respeito aos prazos de postagem; (e) postura ética no AVEA. }\end{array}$
\end{tabular}


Fonte: Elaborado pelas autoras, 2017

Dentro desse contexto, é importante ressaltar que as atividades avaliativas elaboradas e disponibilizadas em AVEA necessitaram ter um caráter problematizador para enriquecerem as discussões. Schmitz (2016), explica que atividades problematizadoras são fundamentais no emprego de metodologias ativas, pois exigem um olhar sobre a realidade, ou pelo menos de parte dela, onde seja possível: (i) construir um problema; (ii) identificar conceitos fundamentais para seu entendimento; (iii) realizar pesquisa para fundamentação, teorização, e apontamento de hipóteses solucionáveis; e aplicação prática. Sendo assim, elaborou-se 10 atividades diferentes, personalizadas para cada grupo, para serem distribuídas e exploradas. A figura 1, representa uma dessas atividades, evidenciando pontos fortes de personalização e problematização, necessários a uma proposta de sala de aula invertida.

Figura 1 - Captura de tela de uma das atividades problematizadoras elaboradas para a Seção "Durante".

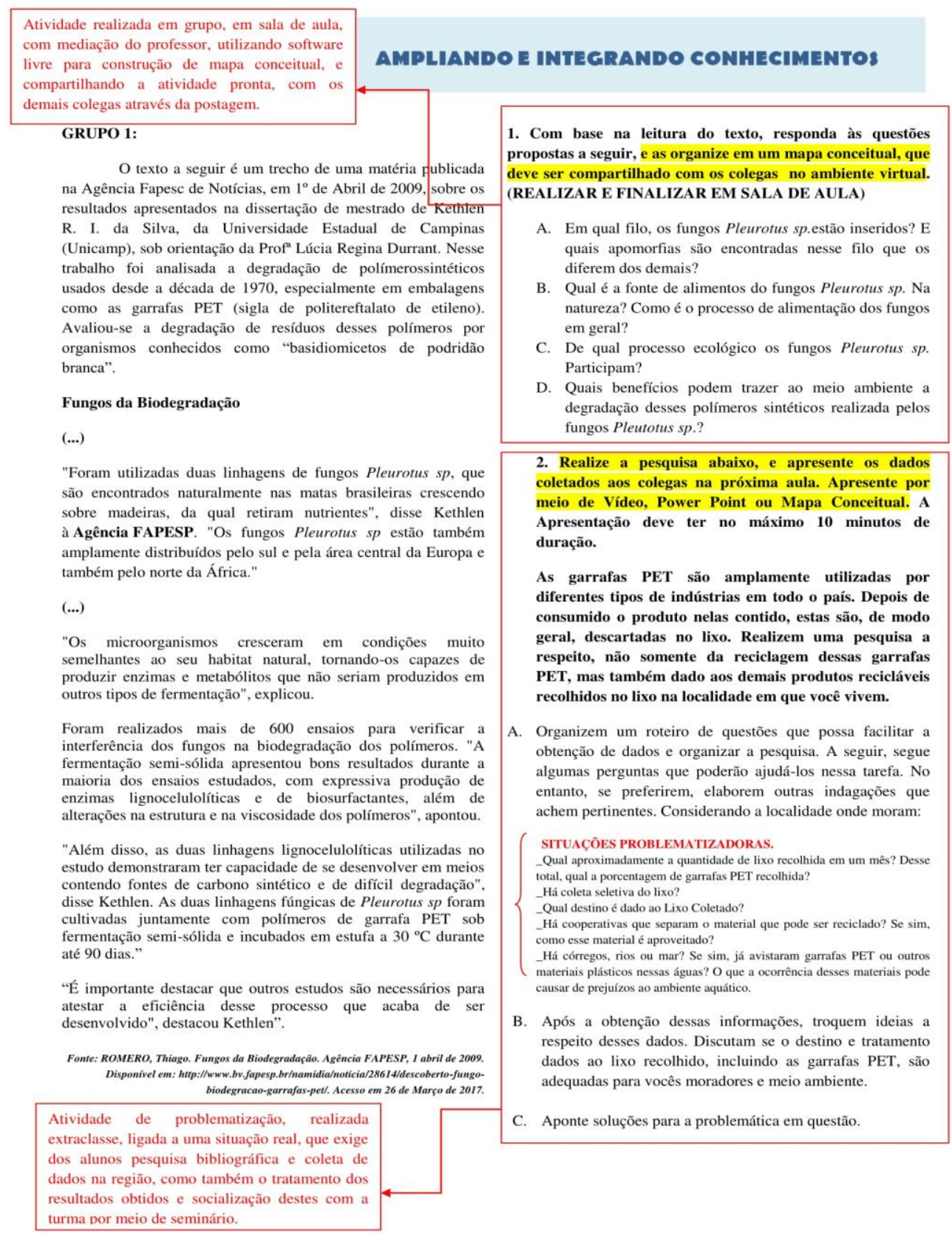

Fonte: Elaborado pelos autores, 2017. 


\subsection{Desenvolvimento do curso no Moodle}

O curso em questão foi construído por uma equipe multidisciplinar, envolvendo profissionais de diferentes áreas do conhecimento, integrando educação e tecnologia. O mesmo pode ser acessado pelo site http://intecedu.ufsc.br, seguindo os tópicos: (a) Cursos; (b) Educação Básica; (c) Instituto Federal de Santa Catarina - IFSC Araranguá; e por fim (d) $4^{\circ}$ ano: Fungos.

Para melhor compreensão do aluno, o curso desenvolvido foi dividido 4 seções, são elas: Orientações; Antes; Durante; Depois. Na primeira seção, como a própria denominação sugere, os alunos eram orientados quanto aos procedimentos metodológicos e encaminhamentos que teriam as aulas referentes ao conteúdo sobre fungos, como visualizado detalhadamente na figura 2.

Figura 2 - Captura de tela mostrando parte da seção "Orientações”.

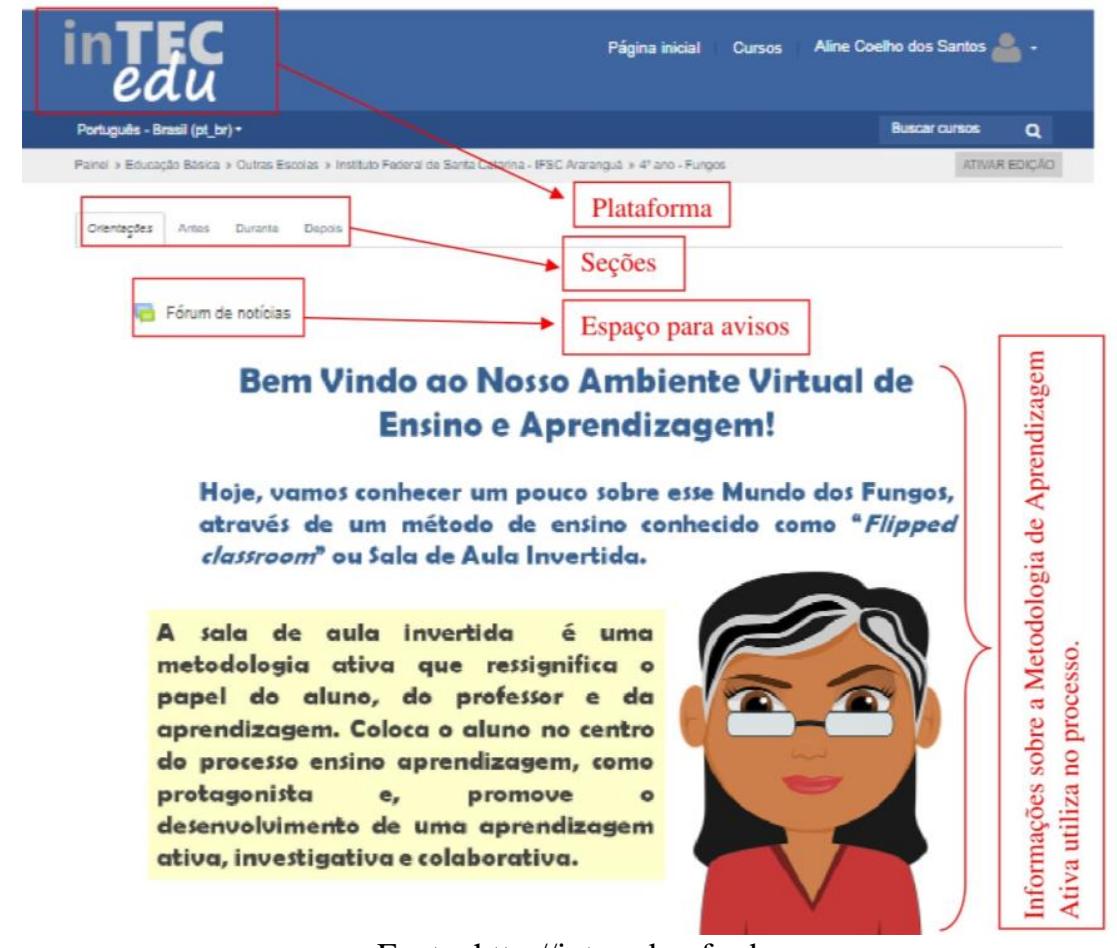

Fonte: http://intecedu.ufsc.br

A segunda seção, intitulada “ANTES”, apresenta-se constituída de materiais para estudo prévio, em diferentes formatos e mídias (mapas conceituais, vídeos curtos, documentários, estudo científico, artigos, site interativo, entre outros) o que caracteriza a personalização do ensino, oportunizada pelo AVEA. Essa seção fora construída para ser explorada na modalidade a distância, antes da ocorrência da aula presencial.

A terceira seção, denominada "DURANTE", foi planejada para ser realizada colaborativamente, em grupo, sendo parte dela realizada em sala de aula, e o restante extraclasse.

Procurando atender aos direcionamentos propostos pela Sala de Aula Invertida, essa seção era composta por atividades problematizadoras elaboradas pela professora titular da disciplina (uma das autoras desse artigo) com temas atuais e relevantes, que levavam os alunos ao desenvolvimento de diferentes produções, como: elaboração de respostas, construção de mapas conceituais, atividade de pesquisa (bibliográfica e pesquisa-ação), e preparação de seminário, como pôde ser observado na figura 1 . 
Por fim, a quarta seção do curso, intitulada "DEPOIS", corresponde ao fechamento do conteúdo em discussão (fungos). Portanto, nela contém a avaliação conceitual, e o questionário para identificação da percepção que os alunos obtiveram com o uso da ferramenta.

\section{Relato sobre desenvolvimento das aulas}

A experiência aqui relatada foi vivenciada no Instituto Federal de Santa Catarina (IFSC), no município de Araranguá, SC, em uma turma de $4^{\circ}$ ano do Ensino Médio Integrado - Vestuário, nas aulas de Biologia. A amostra corresponde a 30 estudantes, com faixa etária entre 15 e 18 anos, sendo 29 estudantes do sexo feminino e somente 1 do sexo masculino.

A aplicação das aulas ocorreu exatamente como programação apresentada na tabela 1. Nesse sentido, o primeiro encontro ocorreu no dia 20 de março de 2017, por meio do desenvolvimento de uma aula conceitual e expositiva, sobre as características gerais dos fungos. Em sequência, foi apresentado aos alunos a plataforma InTecEdu que seria utilizada nas próximas semanas, bem como a metodologia de Sala de Aula Invertida que estaríamos adotando a partir daquele momento. Por fim, os alunos foram orientados a realizarem a inscrição no curso e fazerem o estudo prévio dos materiais propostos na primeira seção.

O segundo encontro presencial, era para ser o momento "chave" da metodologia adotada, caracterizado pela aprendizagem ativa, onde os alunos discutem, colaboram uns com os outros e produzem, pois, entende-se que nesse momento os alunos já deveriam ter estudado previamente os materiais disponibilizados uma semana antes. No entanto, dos 30 alunos participantes, apenas 11 realizaram a matrícula no curso, e tiveram acesso aos materiais. Tal fato prejudicou a dinâmica de sala de aula, e impediu a conclusão em sala da primeira etapa da atividade proposta. Conforme justificativa apresentada pelos alunos não inscritos, o fato de não terem acessado o curso não está associado a falta de acesso a computadores, dispositivos móveis ou internet, mas sim a falta de hábito de estudar previamente e/ou trabalhar em AVEA.

No entanto, apesar desse imprevisto, a aula cumpriu com seu objetivo, como pode ser observado na figura 3, que representa o trabalho de um dos grupos formados para o desenvolvimento da atividade proposta. Na foto é possível identificar pontos fortes sobre o método de sala de aula invertida, como: (a) trabalho colaborativo, em grupo; (b) nenhum dos integrantes do grupo encontra-se ocioso (sem atividades); (c) eles realizam ao mesmo tempo tarefas diferentes; (d) professor na posição de mediador.

A divisão de tarefas apontadas nos itens "b" e "c", é resultado de um primeiro momento, em que todos estudaram a atividade proposta e realizaram pesquisa. Nessas atribuições ficou claro que cada integrante do grupo, optou por realizar atividades nas quais tinham maior interesse ou afinidade. Tal fato, só foi possível porque a atividade proposta, por ser personalizada, já estava planejada para atender diferentes perfis de aprendizagem em uma mesma turma, e permitiu que o aluno se percebesse agente ativo, em algum momento, do processo de ensino-aprendizagem.

Das atividades que estão sendo desenvolvidas, é possível identificar: (i) definição de conceitos e construção do mapa conceitual; (ii) realização de pesquisa em diferentes fontes que pudessem subsidiar a elaboração das respostas; e (iii) elaboração de gráficos, como forma de otimizar a exposição dos dados encontrados. Outro ponto relevante, observado na figura 3 , é a localização do professor dentro da sala de aula como mediador. Com essa posição o professor faz atendimentos individuais nos grupos, auxiliando os alunos nos procedimentos de pesquisa, no uso da ferramenta, e dando 
direcionamentos para a construção de suas conclusões. Percebe-se, que ele sai do espaço frontal da sala de aula e vai para perto do aluno, orientando-os apenas, e não transmitindo o conhecimento pronto.

Figura 3: Aplicação de Sala de Aula Invertida - Seção 2.

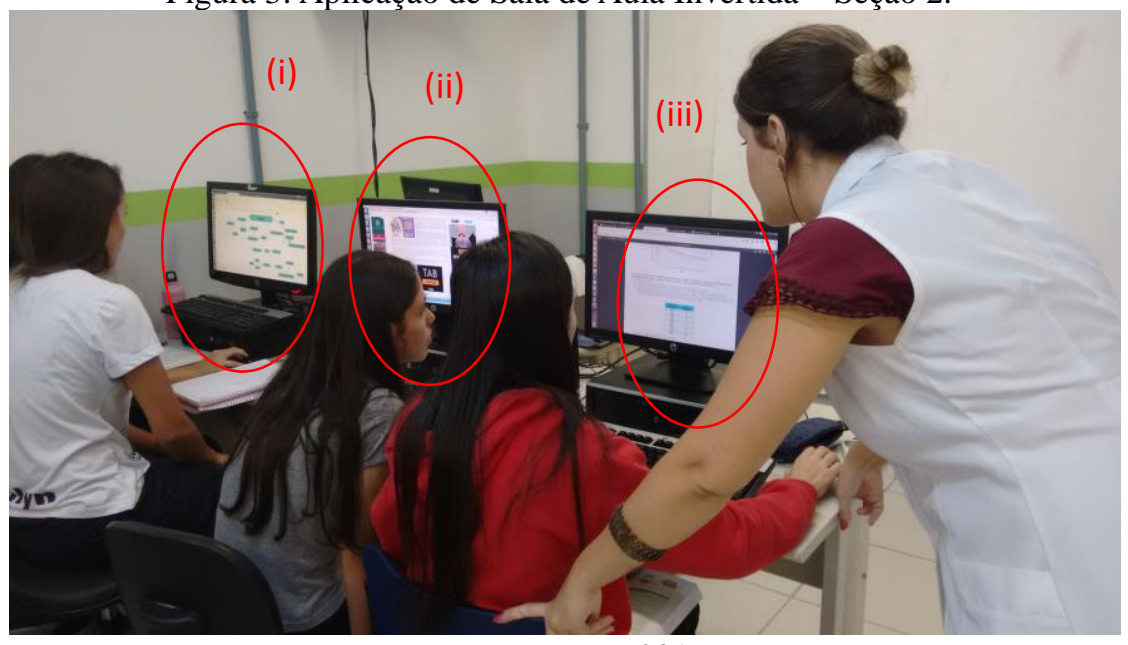

Fonte: autores, 2017

Assim que as atividades foram finalizadas, os alunos postaram suas conclusões no AVEA, e deram segmento a segunda parte do trabalho, aprofundamento da pesquisa, coleta de dados e apresentação de resultados em seminário.

Após as atividades concluídas, os alunos realizaram no AVEA uma avaliação conceitual, com flexibilização de dia e horário para realização da avaliação. Os alunos foram informados previamente de como funcionaria esse sistema, podendo eles, dentro do período de uma semana, escolherem o dia e o horário para realização da avaliação conceitual, sendo assim eles poderiam realizá-la no melhor momento, condizente com seu preparo.

Todos os alunos, sem exceção, realizaram a avaliação e as atividades propostas no AVEA, sanando uma deficiência bem comum no cotidiano escolar, como a necessidade de realizar avaliações de segunda chamada para os faltosos do dia.

Por fim, os alunos foram submetidos a uma avaliação da ferramenta utilizada, identificando pontos que foram positivos e negativos em relação a essa experiência.

\subsection{Pontos positivos e negativos: Um relato dos alunos.}

Conforme relatos apresentados pelos alunos, foi possível identificar alguns pontos fortes e fracos em relação ao uso da ferramenta, como evidenciado na tabela 2 , logo abaixo.

Tabela 2: Relato apresentado pelos alunos quanto ao uso da ferramenta.

PONTOS FORTES

"Excelente ferramenta de aprendizagem, prático de mexer e bem desenvolvido. "

"Ao todo, o Ambiente Virtual de Aprendizagem é uma ótima plataforma de estudos e melhora a qualidade do ensino. "

"Me motivou mais a estudar e a disponibilidade dos conteúdos a qualquer momento facilitou o meu plano de

\section{PONTOS FRACOS}

"É fácil de usar, porém pouco tempo para executar as atividades."

"O ambiente virtual é bom, pois podemos ter acesso aos materiais antes da aula, mas a prova online é complicada porque o computador pode travar no meio dela e a internet cair, o que nos deixa muito nervosa e não fazendo uma boa prova."

"Foi boa a utilização desse site, mas também, fiquei um 

realiza-la. Porém, usando o site me fez ir atrás mais do conteúdo. "

"Maior aproveitamento do tempo de estudos e de recursos visuais, aumento e reforço do que se foi aprendido em sala de aula e inovação."

"Rompimento do modo convencional e incentivo ao estudo."

Fonte: autores, 2017
"Pontos negativos: pouco tempo fornecido para a realização da avaliação final, equivalente ao número de questões. "

Outro ponto interessante encontrado no relado dos alunos, foi o fato de afirmarem não estar acostumados a usarem AVEA em suas atividades e estudos, como pode ser observado nas duas falas a seguir: "É interessante para a interação coletiva. Torna as aulas mais dinâmicas. Mas por conta do costume com as aulas e tarefas presenciais, acabamos esquecendo da plataforma virtual $e$ as atividades aqui propostas."; e "Positivos: Acesso em qualquer lugar, facilita os estudos em casa, incentiva a fazer novas pesquisas, permite download dos arquivos, etc. Negativo: minha memória é horrivel, as vezes esqueço de entrar e sites, mas vou me esforçar.".

\section{Conclusão}

Em linhas gerais, foi possível, nesse trabalho, relatar a construção do curso em AVEA utilizando Sala de Aula Invertida, descrever como ocorreu a execução das aulas, bem como registrar a opinião dos alunos frente ao uso da ferramenta em discussão. Quanto à construção do curso em AVEA - Moodle, foi possível perceber o quanto ele foi apropriado para aplicação da Metodologia de Aprendizagem Ativa, Sala de Aula Invertida, tanto no que condiz a personalização do ambiente necessário para atender as diretrizes propostas pelo método em questão, como também pelo fácil cadastramento dos alunos, e acesso em diferentes dispositivos móveis.

As ferramentas disponibilizadas pelo Moodle, além de fácil entendimento, são muito completas, o que possibilitou a postagem de diferentes mídias e a construção de atividades diversas, que são propostas trazidas pelas metodologias de aprendizagem ativa, como forma de rompimento ao ensino tradicional. Em relação à elaboração das atividades propostas e a aplicação das aulas, dois apontamentos devem ser ressaltados, um em relação as atividades práticas propostas em sala de aula, na seção "durante", e outra sobre o estudo prévio que deve ocorrer na seção "antes".

Quantos as atividades propostas na seção "durante", foi identificado que estas necessariamente precisaram estar contextualizadas e problematizadoras, para que o aluno possa realizar buscas e fazer leituras em diferentes fontes bibliográficas, para então elaborar a resposta com suas conclusões. Do contrário, a exploração torna-se "pobre", e a aula diferencia-se da tradicional apenas pelo recurso tecnológico que está sendo utilizado, pois perguntas diretas e sem contextualização, não exigem grandes buscas por parte do aluno, não aproxima o que está sendo estudado com a realidade, e não exige do aluno reflexão e apontamento de soluções.

Sobre o estudo prévio que deveria ser realizado na seção "antes", e não ocorreu como o planejado, é identificado na própria fala dos alunos, quando estes mencionam não estarem acostumados a utilizarem AVEA. É evidente que toda mudança necessita de um tempo de adaptação, e sendo essa a primeira experiência da turma quanto ao uso de AVEA e Sala de Aula Invertida, fica compreensível essa falha ocorrida no processo. 
Por fim, quanto aos pontos fracos e fortes apontados pelos alunos, nota-se uma percepção dos mesmos quanto a melhoria da aprendizagem, por aumentar a qualidade do estudo e proporcionar novas formas de aprender, em contrapartida surge a necessidade do professor refletir melhor sobre as possibilidades da ferramenta junto ao perfil da turma, para disponibilizar o tempo necessário para execução das atividades e avaliações.

\section{REFERÊNCIAS}

BACICH, Lilian; NETO, Adolfo Tanzi; DE MELLO TREVISANI, Fernando. Ensino híbrido: personalização e tecnologia na educação. Penso Editora, 2015.

DOUGIAMAS, Martin; TAYLOR, Peter. Moodle: Usando Comunidades De Aprendizes Para Criar Um Sistema De fonte Aberta De Gerenciamento De Curso. In: Moodle: Estrategias Pedagogicas e Estudos de caso. Universidade do Estado da Bahia, 2009. p. 15-34.

KENSKI, Vani Moreira. Educação e tecnologias: O novo ritmo da informação. Campinas, SP: Papirus, 2012.

MORAN, José. Ensino e aprendizagem inovadores com tecnologias audiovisuais e telemáticas, In: MORAN, J., MASETTO, M. e BEHRENS, M. Novas tecnologias e mediação pedagógica. Campinas, SP: Papirus, 2000.

PEREIRA, Alice Theresinha Cybis; SCHMITT, Valdenise; DIAS, M. R. A. C. Ambientes virtuais de aprendizagem. AVA-Ambientes Virtuais de Aprendizagem em Diferentes Contextos. Rio de Janeiro: Editora Ciência Moderna Ltda, p. 4-22, 2007.

REXLAB. Programa InTecEdu. Disponível em: <http://rexlab.ufsc.br/projects/intecedu>. Acesso em: 27 de setembro de 2017.

SCHMITZ, Elieser Xisto da Silva. Sala de Aula Invertida: Uma abordagem para combinar metodologias ativas e engajar alunos no processo de ensinoaprendizagem. 2016. 187 p. Dissertação (Mestrado) - Curso de Mestre em Tecnologias Educacionais em Rede, Universidade Federal de Santa Maria, Santa Maria, 2016.

VALENTE, José Armando. Prefácio. In: Blended learning e as mudanças no ensino superior: a proposta da sala de aula invertida. Educar em Revista, n. 4, 2014.

VALENTE, José Armando. Blended learning e as mudanças no ensino superior: a proposta da sala de aula invertida. Educar em Revista, n. 4, 2014.

VALENTE, Luís; MOREIRA, Paulo. Moodle: moda, mania ou inovação na formação?Testemunhos do Centro de Competência da Universidade do Minho. In: Actas da V Conferência Internacional de Tecnologias de Informação e Comunicação na Educação-Challenges. 2007. p. 781-790. 